\title{
247 THE FULLY HUMAN ANTIBODY SRF617 IS A POTENT INHIBITOR OF ECTO-ENZYME CD39 IN VIVO
}

'Stephan Matissek*, ${ }^{1}$ Marisella Panduro Sicheva, ${ }^{1}$ Secil Koseoglu, ${ }^{1}$ Ricard Masia, ${ }^{1}$ Michael Warren, ${ }^{1}$ Matthew Rausch, ${ }^{1}$ Benjamin Lee, ${ }^{2}$ Sandra Pommey, ${ }^{2}$ Isabelle Cousineau, ${ }^{2} J$ ohn Stagg, 'Vito Palombella, ${ }^{1}$ Andrew Lake. 'Surface Oncology, Inc., Cambridge, MA, USA; ${ }^{2}$ Université de Montréal, Montreal, Canada

Background The purine nucleotide adenosine plays an important role in dampening both the innate and adaptive arms of the immune system. In contrast, extracellular adenosine triphosphate (ATP), which can be generated at high levels due to cell stress, initiates proinflammatory responses. Extracellular ATP can be produced in cancerous tumors, and its conversion to adenosine monophosphate by CD39 (ENTPD1) and subsequent degradation to free adenosine by CD73 (NT5E) in the tumor microenvironment (TME) is a major contributor to tumor immune evasion. Accordingly, CD39 serves as an important immune regulator. CD39 is highly expressed in the TME of various tumor types, including lung, prostate, colon, ovarian, and pancreatic cancers, making CD39 an attractive target for immune-based anticancer therapy.SRF617 is a fully human anti-CD39 antibody designed to target human CD39 and inhibit its enzymatic activity. SRF617 provides a dual mechanism of immune activation by increasing inflammatory responses through accumulation of free ATP and alleviating immune suppression by reducing adenosine production. In this way, SRF617 shifts the TME to a proinflammatory state leading to reactivation of the immune system and antitumor function.

Methods A pharmacokinetic/pharmacodynamic profile was determined in CT26 tumor-bearing human CD39 knock-in (hCD39 KI) mice by measuring plasma SRF617 concentration, peripheral blood target occupancy (TO), and ATP hydrolysis activity in frozen tissue sections. Wild-type mice bearing subcutaneous (s.c.) KPC1245 tumors were treated with anti-murine CD39 antibody, alone or in combination with gemcitabine. Results Circulating SRF617 was observed in the plasma after intravenous injection of CT26 tumor-bearing hCD39 KI mice, which correlated with CD39-bound SRF617 on B cells. The presence of SRF617 correlated with a decrease in ATPase activity and CD39 expression in tissues. In the KPC tumor model, a combination effect was observed for anti-CD39 treatment with gemcitabine. Treatment with anti-CD39 antibody resulted in an increase in CD8 $+\mathrm{T}$ cells in the TME as measured by flow cytometry.

Conclusions SRF617 treatment of hCD39 KI mice led to reduction of ATPase activity in the TME that correlated with peripheral TO and plasma concentration. Anti-CD39 treatment alone or in combination with gemcitabine led to tumor growth inhibition and increased tumor-infiltrating lymphocytes as measured by flow cytometry in s.c. KPC tumors. These studies demonstrate the therapeutic potential of targeting CD39 for the treatment of cancer. SRF617 is currently being evaluated in a Phase 1 clinical trial in patients with advanced solid tumors (NCT04336098) and in combination with chemotherapy in patients with pancreatic cancer.

http://dx.doi.org/10.1136/jitc-2021-SITC2021.247 